Artículo de Revisión

\title{
OBSTÁCULOS Y RETOS PARA EL DESARROLLO DE SISTEMAS DE INFORMACIÓN EN EL SECTOR SALUD
}

\section{OBSTACLES AND CHALLENGES FOR THE DEVELOPMENT OF INFORMATION SYSTEMS IN THE HEALTH SECTOR}

\author{
Agustín F. Gutiérrez T. ${ }^{1}$, Ricardo Peña G. ${ }^{2}$, Norma I Peña G. ${ }^{3}$, Roberto Rosario Cruz ${ }^{4}$, Saúl López Silva ${ }^{5}$ \\ Recibido para publicación: Octubre 18 de 2017 - Aprobado para publicación: Diciembre 13 de 2017
}

\begin{abstract}
RESUMEN
Objetivo. El propósito de los Sistemas de Información en de la Salud (SIS) es mejorar la salud de los individuos y las poblaciones mediante la aplicación apropiada del conocimiento generado por la organización de la información en dichos sistemas. Materiales y métodos. Se realizó una revisión sistemática de literatura a través de las experiencias y lineamientos en el desarrollo y la implementación de un SIS. Resultados. El análisis de los requisitos es el punto de partida para el desarrollo o la adquisición de cualquier producto de software. El SIS debe contemplar la inclusión de los datos necesarios para la investigación y permitir su organización dependiendo del tipo de investigación que se quiera realizar; debe simplificar y acelerar el acceso a la información crítica al mismo tiempo que controla el acceso a los datos; debe facilitar el tratamiento estadístico de grandes cantidades de datos; $y$, finalmente, debe facilitar el intercambio de datos entre diversos sistemas. Conclusiones. El conjunto de los obstáculos y retos para el desarrollo de los SIS enumerados en este artículo confirma la gran complejidad que presenta este tipo de sistemas de información, para ello se requiere de una planeación madura y detallada.
\end{abstract}

Palabras clave: sistemas, información, salud, obstáculos, retos.

\begin{abstract}
Objective. The purpose of Information Systems in Health (SIS) is to improve the health of individuals and populations through the appropriate application of knowledge generated by the organization of information in such systems. Materials and methods. A literature review was conducted through the experiences and results obtained in the development and implementation of an SIS. Results. The analysis of the requirements is the starting point for the development or acquisition of any software product. The SIS must consider the inclusion of the necessary data for the investigation and allow for its organization depending on the type of inquiry that will be carried out. It must simplify and accelerate access to critical information while controlling access to data, facilitate the statistical processing of large amounts of data, and finally facilitate the exchange of data between different systems. Conclusions. The set of obstacles and challenges for the development of the SIS listed in this article confirms the high complexity of this type of information systems for which mature and detailed planning is needed.
\end{abstract}

Keywords: systems, information, health, obstacles, challenges.

\footnotetext{
${ }^{1} \mathrm{PhD}$., Universidad Autónoma de Guerrero -UAGro, Profesor Investigador, Unidad Académica de Ciencias y Tecnologías de la Información -UACyT. Correspondencia: afgutierrezt@uagro.mx

${ }^{2}$ M.Sc Profesor Investigador, Unidad Académica de Ciencias y Tecnologías de la Información -UACyT,

${ }^{3}$ C.PhD. , Universidad Autónoma de Guerrero -UAGro, Directora, Unidad Académica de Ciencias y Tecnologías de la Información UACYTI.

${ }^{4}$ M.Sc., Universidad Autónoma de Guerrero -UAGro, Profesor Investigador, Unidad Académica de Ciencias y Tecnologías de la Información -UACyTI.

${ }^{5} \mathrm{PhD}$., Universidad Autónoma de Guerrero -UAGro, Coordinador de Posgrado, Facultad de Medicina.
} 


\section{INTRODUCCIÓN}

El propósito de los sistemas de información en el ámbito sanitario (SIS) es mejorar la salud de los individuos y las poblaciones mediante la aplicación apropiada del conocimiento generado por la organización de la información en dichos sistemas. Este proceso incrementa la calidad de los datos, así como la eficiencia en el acceso ala información almacenada $(1,2,3)$.

Sin embargo, a la fecha aún existen muchos problemas por resolver antes de alcanzar el ideal de contar con sistemas que permitan acceder y tratar eficazmente la información desde cualquier lugar y por diferentes medios.

Elanálisis de los requisitosesel puntodepartida para el desarrollo o la adquisición de cualquier producto de software. Esta tarea se basa en la precisión de las necesidades funcionales que solicita explicitamente el cliente (o usuario), pero tambièn por del establecimiento de requerimientos implicitos que debe poseer cualquier sistema informático desarrolladoprofesionalmente para completar su calidad técnica. $(4,5)$.

Es por ello que el conocimiento de los obstáculosy retos que suelen presentarse como eventos o actividades riesgosas puede y debe mejorar los criterios de elaboración o compra de un producto de software, especialmente en un sector tan sensible como el de la salud.

En este trabajo se presenta un conjunto de situaciones con estas características, las cuales se proponen comosituaciones condicionantes de los requerimientos de unSIS.

\section{MATERIALES Y MÉTODOS}

En la realización de este trabajo se hizo una revisión sistémica de la literatura relacionada con las experiencias y resultados obtenidos en el desarrollo y la implementación de un SIS, y con los lineamientos y recomendaciones internacionales para el desarrollo y la implementación de un SIS. Para ello se llevaron a cabo tres etapas:

En la primera etapa, se realizó la organización de la información precedida de la consulta de libros, revistas de divulgación o de investigación científica, sitios Web y demás informaciónnecesariaparainiciar labúsqueda. La información se organizó por relevancia, distinguiendo los principales documentos de los secundarios, se definió una estructura organizativa determinada de los datos para hacer una presentación concisa de los mismos. Las variables o características se limitan a los criterios escogidos por el autor (6). En la segunda fase se seleccionaron los estudios que cumplieron con los criterios y así mismo, se excluyeron los que no fueron relevantes para el objetivo de la revisión. Finalmente, en la tercera etapa se analizó la información ya organizada, profundizandosobre los documentos másútiles para la temática del estudio.

\section{RESULTADOS}

De acuerdo con los objetivos planteados y la revisión bibliográfica realizada, en el contexto de los SIS, se ha identificado un conjunto de más de quinientas funciones propias las cuales se engloban en las siguientes:

\section{A) Requerimientos generales}

El sistema debe contar con una estructura modular y debe posibilitar que el expediente del paciente sea compatible con otros sistemas. También debe ser posible el acceso simultáneo a los registros del expediente, así como el acceso al plan de tratamiento del paciente. Finalmente, el sistema debe incluir tutoriales para el entrenamiento del usuario.

\section{B) Interfaz de usuario}

El sistema debe hacer uso de una interfaz gráfica que trabaje a través de la administración de ventanas, debido a que esto es algo 
conocido para la mayoría de los usuarios y les facilita la comprensión del funcionamiento del sistema. Además, debe incluir funciones tales como accesos directos (atajos), despliegue de información global o despliegue selectivo, mensajes del sistema (especialmente, funciones de advertencia), grabado de datos clínicos por diferentes medios, procesamiento de palabras y reorganización gráfica decontenidos.

\section{C) Captura de datos}

Debe incluirse la utilización de diversas herramientas de captura (diferentes formatos y medios), tales como: teclado, escáner, código de barras, tarjetas inteligentes, tarjetas ópticas, voz, huellas digitales, etc. Asimismo, los datos capturados deben incluir información obligatoria, valores por omisión y predefinidos, advertencias de haber excedido los rangos. Finalmente, debe ser posible que el usuario diseñe las plantillas y formatos que necesite.

\section{D) Funciones de soporte}

Las bases de datos de los diversos módulos del sistemas deben ser compartidas, y el sistema debe incluir reconocimiento de textos, grabado automático de acciones estándares (como recetas, por ejemplo), tratamiento de pacientes anónimos (sin número de identificación personal), manejo de números de identificación alternativos y actualización de datos de identificación personal, entreotros.

\section{E) Seguridad y confidencialidad}

El sistema debe cumplir con los requerimientos legales comunes, y debe incluir: notas (relacionadas con hipersensibilidad del paciente ante ciertos medicamentos, listas de medicamentos descontinuados, etc.), control de acceso e identificación y autenticación de la identificación, clasificación de la información (por niveles de seguridad) y del acceso (niveles de acceso), control de conexión/desconexión para cada acción (tiempo, acción realizada, etc.), protección para la comunicación externa, protección para la integridad de los datos y todas aquellas me- didas que garanticen la fiabilidad del sistema. Para lo anterior, el sistema incluye procesos que aseguran la seguridad física, la autenticación de datos y de usuarios, el cifrado, la firma digital, las claves de control de acceso, etc.

\section{F) Funciones de salidas}

El sistema debe generar reportes globales y estándares, permitir la "exportación" de datos y el control de impresión, impresión de etiquetas y códigos, función de archivo, dar seguimiento a las funciones de salida, asegurar la calidad de las salidas y, en general, proporcionar medidas que permitan evaluar el logro de objetivos.

\section{G) Comunicación}

Elsistemadebeactuarcomounsistemadesoporte de decisiones, permitiendo la comunicación con otros sistemas y el almacenamiento de imágenes médicas de pacientes individuales, así como presentando plantillas para las diferentes partes del cuerpo y todas aquellas aplicaciones multimedia que faciliten la toma de decisiones.

\section{D) Documentación del sistema}

Aquella que sea necesaria para el adecuado uso y operaciòn de las aplicaciones incluìdas en el sistema entre una gama que abarca los documentos siguiente: tutoriales, instrucciones de instalación, manuales de usuario, solución deproblemas, manual de referencia, funciones de ayuda, procedimientos de operación, documentación técnica, modelo de datos, protocolos de procedimiento y tratamientos.

\section{E) Estructura del expediente}

Como parte importante del sistema de información de la salud, el expediente debe hacer uso de palabras clave y terminologías, así como contar con una base de datos de terminología nacional, posibilidad de intercambiar información entre el módulo de expediente de pacientes y el módulo administrativo, documentar la acción de enfermería, crear notas sin visitas del paciente y utilizar sistemas de clasificación /codificación. 


\section{F) Requerimientos especiales para la actividad paramédica}

El sistema debe posibilitar el seguimiento y aseguramiento de la calidad, así como la evaluación y logro de los objetivos la actividad paramédica, además de posibilitar el acceso a los programas predefinidos de cuidado y rehabilitación, administración del equipo de rehabilitación, soportepara el cuidadoencasa, estándares de clasificación de intervencionesy procedimientos, etc.

\section{G) Requerimientos especiales para la actividad investigativa}

El SIS debe contemplar la inclusión de los datos necesarios para la investigación y permitir su organización dependiendo del tipo de investigación que se quiera realizar; debe simplificary acelerar el acceso a la información crítica al mismo tiempo que controla el acceso a los datos; debe facilitar el tratamiento estadístico de grandes cantidades de datos; $y$, finalmente, debe facilitar el intercambio de datos entre diversos sistemas.

En algunas organizaciones puede ser conveniente almacenar los datos necesarios para la investigación (derivados de los datos clínicos) en un lugar diferente al utilizado para propósitos clínicos. La existencia de almacenes de datos diferentes hace necesaria, sin embargo, la creación de un "mediador" o "traductor" entre almacenes, locual implica la utilización de estándares para la comunicación, especialmente cuando estos "mediadores" pertenecen a erdifentes instituciones.

\section{DISCUSIÓN}

Los obstáculos y retos identificados son los siguientes y pueden clasificarse o tipificarse en los grupos que a continuación se enuncian.

\section{A) Seguridad:}

Además de la protección física de los equipos para evitar daños con la consecuentepérdida de la información, los SIS en este rubro, deben instrumentar controles que garanticen los siguientes apectos:

1) la integridad, evitar la alteración accidental o premeditada de la información;

2) el acceso, solamente el personal autorizado (auenticado) podrá acceder a la información pertinente $y$

3) la confidencialidad, evitar la divulgación no autorizada de la información.

Para definir niveles de seguridad se hace necesario llevar a cabo acciones como: la definicón de roles y sus funciones asociadas y de los niveles de acceso a la información correspondientes; el establecimiento de reglas y/o barreras para la divulgación de la información y la educación de los usuarios sobre la necesidad y pertinencia de la confidencialidad de los datos, ya quela mayoría de las fallas de seguridad se relacionan con la falta o mala ejecución de los procedimientos, 0 la mala intención del elemento humano $(6,7)$.

Por todo lo anterior, es necesario contar con un plan de prevención que permita recuperar los datos en caso de "desastre".

\section{B) Normalización:}

No siempre se cuenta o se tiene disposición de instrumentos reguladores reconocidos nacional o internacional. En el ámbito de los SIS se trabaja al menos con los siete tipos de normas que a continuación se enuncian.

1) Normas del identificador: que guían el trabajo de creación de identificadores universales para el área de la salud.

2) Normas de comunicación: establecen o definen los formatos de los mensajes. Entre estos se encuentra el estándar DICOM para la comunicación de imágenes digitales que ha sido incorporado en los sistemas para el tratamiento de imágenes europeos y japoneses (8) y el estándar HL7 (Nivel de salud 7) para la transmisión de datos como información de pacientes, imágenes, resultados de laboratorio, etc. (9) 
3) Normas de contenido y estructura: como los propuestos por la American Society for Testing and Materials (ASTM), entre las que se encuentran la ASTM E1633, Standard Specification for Coded Values Used in the Electronic Health Record (2007), la ASTM E1239, Standard Practice for Description of Reservation/RegistrationAdmission, Discharge, Transfer (R-ADT) Systems for Electronic Health Record (EHR) Systems (2004) y la ASTM E1715 Standard Practice forAn Object-Oriented Model for Registration, Admitting, Discharge, and Transfer (RADT) Functions in ComputerBased Patient Record Systems (2017). (10)

4) Representaciones de datos clínicos (códigos): se utilizan para documentar diagnósticos y procedimientos. Incluyen entre otros el estándar para la clasificación de enfermedades o International Classification of Deseases (ICD), ahora en versión 10 (ICD10) del 2016 de la Organización Mundial de la Salud (OMS-WHO) (11); las nomenclaturas sistematizadas de la medicina humana y veterinaria o Systematized Nomenclature of Medicine-Clinical Terms (SNOMED-CT)) de la International Health Terminology Standars Development Organization (IHTSDO) (12, 13); los nombres y códigos de identificación para observaciones de laboratorio o Logical Observation Identifiers Names and Codes (LOINC) del Regenstrief Institute (14) y la Nomenclatura Médica Gabrieli (GMN), para representar las frases médicas en su forma canónica o vernácula. (15)

5) Normas para la Seguridad de la Información: procedimientos establecidos siguiendo estándares internacionales o propios.

6) Indicadores de calidad: Se trata de la definición y adopción de métricas $y$ procedimietnos que permitan la evalución de los productos o servicios utilizados o brindados en el ámbito de la salud. Su implementación se ve limitada por la falta de acuerdo en los criterios para la medición de la calidad en salud(16).
7) Otros estándares nacionales o internacionales: Por ejemplo aquellos de la Organización Internacional de Normalización (ISO) relacionados directa o indirectamente con la salud (17).

\section{C) Aplicabilidad a la investigación}

Una de las funciones asociadas a las instituciones de la salud que permanece aún a veces olvidada dentro de la aplicación de tecnologías informáticas es aquella de la investigación. La Association of American Medical Collleges (AAMC), considera que las tecnologías de información utilizadas en la mayoría de las escuelas demedicinay hospitales delosEstados Unidosnotomanencuentalasnecesidades dela investigación clínica, debido a que los sistemas de información existentes en el mercado están creado para cubrir las necesidades de los administradores de los hospitales más que las de los investigadores (18).

Así, los SIS deben contemplar la inclusión de los datos necesarios para la investigación y permitir su organización dependiendo del tipo de investigación que se quiera realizar; debe simplificary acelerar el acceso a la información crítica al mismo tiempo que controla el acceso a los datos; debe facilitar el tratamiento estadístico de grandes cantidades de datos; $y$, finalmente, debe facilitar el intercambio de datos entre diversos sistemas.

Esta misma institución establece que la investigación clínica necesita de grandes cantidades de información que debe ser procesada de formas diferentes dependiendo del tipo de investigación clínica de que se trate e identifica cuatro tipos de investigación clínica, las cuales se describen en la Tabla 1.

\section{D) Aspectos legales}

Otro problema es la necesidad de considerar la reglamentación vigente de inviolabilidad. Actualmente ni el sistema ni su administrador tienen responsabilidad legal alguna por las deci- 
siones tomadas por el médico ante un paciente. Aún más, las grandes compañías que actualmente trabajan en el desarrollo de sistemas de la salud siempre añaden como cláusula a sus sistemas la aclaración de que no se responsabilizan ante cualquier consecuencia negativa derivada de la utilización de sus programas.

Una propuesta relacionada con la legalidad de los SIS se basa en el hecho de que en el caso de otros documentos legales importantes (tales como escrituras, testamentos, etc.) se recurre a terceros legalmente reconocidos (por ejemplo, los notarios públicos). La propuesta consiste en crear en forma periódica copias del SIS que serán registradas oficialmente ante un notario para su resguardo. Ante un juicio, el notario podría cotejar su copia contra la de la institución y, por tanto, aseguraría que ésta no ha alterado el sistema a su favor.

\section{E) Riesgos}

El riesgo se concibe como la probabilidad de que una circunstancia adversa ocurra otambién como un ente agente de cambio, como una elección e incertidumbre que acompaña a una decisión. Si los riesgos se hacen realidad, el proyecto se ve amenazado debido a quesu ejecución se retrasa, los costos aumentan y, seguramente, se presentan problemas con el personal.

El análisis de riesgo en ingeniería de software implica, por tanto, plantearnos la pregunta: ¿qué riesgos podrían hacer que nuestro proyecto fracase? Esto permite anticipar los problemas que podrían perjudicar al desarrollo del proyecto o la calidad del producto de software y plantear las acciones necesarias para evitar dichos riesgos o minimizar sus efectos sobre el proyecto o sobre el producto.

Tabla 1. Necesidades de la Investigación clínica

\begin{tabular}{|c|c|c|}
\hline $\begin{array}{l}\text { Tipo de Investigación } \\
\text { Clínica }\end{array}$ & $\begin{array}{l}\text { Requerimientos de } \\
\text { Información }\end{array}$ & $\begin{array}{l}\text { Limitantes } \\
\text { (Requerimientos que no han sido cubiertos completamente) }\end{array}$ \\
\hline $\begin{array}{l}\text { Investigación basada } \\
\text { en la población (19, } \\
\text { 20) }\end{array}$ & $\begin{array}{l}\text { Necesita gran cantidad } \\
\text { de datos, recolectados a } \\
\text { través del tiempo, relativos } \\
\text { a la salud de la población } \\
\text { en general o de un grupo } \\
\text { poblacional. }\end{array}$ & $\begin{array}{l}\text { Estandarización en códigos, vocabularioy nomenclaturas. } \\
\text { Estandarización en la transmisión de datos } \\
\text { Infraestructura para incluir, almacenar y extraer datos. } \\
\text { Estándar para la validación de los datos contenidos en los temas } \\
\text { electrónicos. } \\
\text { Uso de algoritmos que aseguren la validez de los datos }\end{array}$ \\
\hline $\begin{array}{l}\text { In v estigación } \\
\text { traslacional }(21,22)\end{array}$ & $\begin{array}{l}\text { Necesita gran cantidad de } \\
\text { datos relativos a la salud de } \\
\text { un individuo o un pequeño } \\
\text { grupo de individuos en } \\
\text { específico y su entorno. }\end{array}$ & $\begin{array}{l}\text { Instalaciones con un medioambiente controlado donde sea posible } \\
\text { la recolección de datos en tiempo real. } \\
\text { Nomenclatura común para los parámetros clínicos. } \\
\text { Bases de datosy programas que identifiquen los sujetos potenciales } \\
\text { para la investigación. } \\
\text { Tecnologías que tomen en cuenta el creciente número de requisitos } \\
\text { relativos a la investigación clínica. }\end{array}$ \\
\hline $\begin{array}{l}\text { Investigación genética } \\
(20,23)\end{array}$ & $\begin{array}{l}\text { Necesita gran cantidad } \\
\text { de datos, tanto de } \\
\text { individuos específicos } \\
\text { y sus familias, como de } \\
\text { grupos poblacionales } \\
\text { determinados. }\end{array}$ & $\begin{array}{l}\text { Bases de datos clínicos que utilicen un vocabulario controlado para } \\
\text { describir el fenotipo de grandes grupos de individuosy que se deriven } \\
\text { de datos recolectados durante las consultas médicas de rutina. } \\
\text { Acceso al ADN de una gran cantidad de pacientes que puedan ser } \\
\text { utilizados en estudios de asociación o relación genética. } \\
\text { Herramientas bioinformáticas que ayuden a identificar aspectos } \\
\text { genéticos relacionados con la salud y la enfermedad. } \\
\text { Mecanismos que posibiliten la realización de estudios de largo } \\
\text { alcance ntegrando datos genéticos. } \\
\text { Herramientas que permitan guardan y analizar la historia familiary } \\
\text { las pruebas genéticas de los pacientes (perfiles genéticos). }\end{array}$ \\
\hline
\end{tabular}


Arbitraje médico (24, Necesita grancantidad de $25,26)$ datos relacionados con casos específicos
Metodologías para identificar sujetos potenciales para juicios

clínicos, acordes con las regulaciones pertinentes.

Mecanismos que permitan la recolección,organización y

reutilización de datos a lo largo de un juicio.

Terminología estándar.

Fuente: Peña Galeana, R. 2003 (29).

A pesar de que existen riesgos generales y comunes, lostipos deriesgoque pueden afectar a un proyecto dependen de las características de éste y de la organización donde se esté desarrollando, por lo que una tipología general no puede ser exhaustiva. Los factores de riesgo más comunes en la actividad informática se muestran en la Tabla $2(5,27)$.
El incluir el análisis de riesgos dentro del plan de proyecto implica, entonces, que debemos identificarlos, valorar sus consecuencias, establecer controles para evitarlos o minimizarlos y elaborar un plan para su constante supervisión.

Tabla 2. Factores de riesgo

\begin{tabular}{|c|c|}
\hline FACTOR DE RIESGO & RIESGO POSIBLE \\
\hline Tecnología & - La tecnología fundamental (base para el sistema) se debe sustituir por nueva tecnología. \\
\hline Personas & $\begin{array}{l}\text { - Imposibilidad de reclutar personal con experiencia o habilidad. } \\
\text { - Excesiva rotación de personal. } \\
\text { - El personal con experiencia no está disponible en momentos críticos o abandona el proyecto } \\
\text { antes de que finalice. } \\
\text { - La capacitación necesaria para el personal no está disponible. }\end{array}$ \\
\hline Organización & $\begin{array}{l}\text { - Cambio de administración. La nueva administración tiene diferentes prioridades u objetivos. } \\
\text { - La organización enfrenta problemas financieros que reducen el presupuesto del proyecto. } \\
\text { - Un producto competitivo se pone a la venta en el mercado antes de que el sistema se complete. }\end{array}$ \\
\hline Herramientas & $\begin{array}{l}\text { - El hardware esencial no será entregado a tiempo, no está disponible en el mercado o no es } \\
\text { accesible para la organización. } \\
\text { - Las herramientas de auxilio computacional CASE (Computer Aided Software Engineering) son } \\
\text { insuficientes o no tienen el desempeño anticipado. }\end{array}$ \\
\hline Requerimientos & $\begin{array}{l}\text { - Hay más cambios de requerimientos de lo anticipado debido a fallas en el análisis, cambios } \\
\text { en la organización o en su entorno. } \\
\text { - Los clientes no comprenden el impacto de un cambio en los requerimientos. }\end{array}$ \\
\hline Estimación & $\begin{array}{l}\text { - Se subestimó el tiempo requerido para desarrollar el software o el tamaño de éste. } \\
\text { - Las especificaciones esenciales no estarán a tiempo. } \\
\text { - Se subestimó el porcentaje de defectos. } \\
\text { - Se subestimó el costo del proyecto. }\end{array}$ \\
\hline
\end{tabular}

Fuente: Peña Galeana, R. 2003 (29). 


\section{F) Otros problemas}

A continuación se mencionan algunos otros problemas relacionados con los SIS que tienen que ver con consideraciones socioeconómicas asociadas a su utilización (28).

1. Si el SIS es una serie cronológica de datos, entonces debe preservar esta estructura, presentando la información contenida en forma cronológica. Este es el caso de los expedientes clinicos. Desafortunadamente, hasta la fecha no se ha desarrollado ninguno que cumpla con esta regla. La razón principal es que es difícil crear una estructura adecuada a las necesidades de todas las especialidades médicas, que presente los datos en secuacial y cronológicamente y además trabaje en ambiente "de ventanas" alcual estamosacostumbrados losusuarios.

2. Existe un concenso general en que los SIS en el futuro, se conserven almacenados en medios móviles y en poder del paciente (28). Lo anterior permitirá su atención en cualquier momento y lugar del mundo, pero presenta dos problemas principales: a) para que realmente sea posible utilizar el un SIS en cualquier lugar del mundo, éste debe de haberse desarrollado y mantenerse en forma estandarizada; y b) el hecho de que el SIS se almacene en medios móviles incrementa la posibilidad de manipulación, robo o extravío, lo que implica disminución de la confiabilidad y confidencialidad de la información.

1. Entre otros, la $A A M C$ plantea que, dada la inevitable obsolescencia de la tecnología computacional, los SIS corren el riesgo de convertirse en potencialmente inutilizables o ininteligibles, y de no poder funcionaren ambiente computacionales más modernos. Se recomienda retener grandes volúmenes de datos por poco tiempo, reduciendo el nivel de detalle de aquellos cuando sea posible conservar su significado y su valor (por ejemplo, reduciendo la definición de las imágenes o la cantidad demuestras) o pasándolos a otro medio de conservación cuando pierdan su validez o caducan por el tiempo. (2)
2. El uso del SIS trae como consecuencia que se modifiquen, agreguen o desaparezcan algunas actividades y puestos. Por ejemplo, la actividad realizada por el personal de archivo cambia y posiblemente desaparece el puesto de mensajero, mientras que aparece el puesto de administrador de red. Lo anterior lleva a los siguientes problemas:

a. El uso del SIS puede generar desempleo, al implicar el despido de algunas personas. Lo anterior podría solucionarse mediante la capacitación del personal, lo cual le posibilita la transferencia a los nuevos puestos.

b. De acuerdo con la AAMC, el éxito de dichos SIS (y, en general, de cualquier Tecnología de la Información utilizada en este ambiente), depende mucho del factor humano involucrado, que en muchas ocasiones se resiste a utilizar la tecnología o los estándares asociados a ella. Así, es posible predecir que muchos profesionales de la salud serán incapaces de adaptarse a las nuevas tecnologías, por lo que no solo habrá personas cuyos puestos desaparezcan y que no serán reabsorbidas por la organización, sino que también habrá profesionales cuya resistencia a utilizar el SIS ocasione problemas laborales para la organización.

3. Si tomamos en cuenta que tradicionalmente el médico es visto por el paciente bajoun filtro de reverencia y respeto, el uso de un SIS (especialmente si éste contiene ligas a sugerencias y ayudas para el diagnóstico y tratamiento) puede ocasionar desconfianza en el paciente acerca de la capacidad del médico y menoscabar su imagen ante la comunidad, lo cual a su vez podría restar confianza en sí mismo al personal médico.

4. Finalmente, el uso de los SIS puede implicar la despersonalización de las relaciones humanas dentro de la organización, tanto entre médicos y pacientes como entre el mismo personal de la salud. 


\section{CONCLUSIONES}

El análisis de los obstáculos y retos para el desarrollo de los SIS enumerados en este artículo confirma la gran complejidad que presenta este tipo de sistemas de información. Su conocimiento nos permite el poderlos tomar en consideración para evitar consecuencias trágicas para sus usuarios, pacientes, administradores y personal de la salud.

Los resultados del proceso de revisión documental presentados demuestran que el éxito en el desarrollo e implementación de SIS requieren de una planeación madura y detallada.

Finalmente, cabe mencionar que dada la diversidad de funciones a contemplar y dado el rápido ritmo de cambio tecnológico en la materia, aún queda mucho por hacer para definir un modelo de procesos y una metodología de desarrolo de proyectos o de aquisición de productos de software adecuados a la realidad circundante en relación a los SIS.

La magnitud de la tarea, sin embargo, no debe verse como un obstáculo sino como un reto que impulse su ejecución teórico-práctica. De así hacerlo, se logrará una base y se dará el impulso necesario para que la modernización y mejora de los sistemas de información de todas las organizaciones de la salud sea, no solo un sueño, sino una realidad.

\section{CONFLICTO DE INTERÉS}

Los autores declaran no tener conflicto de interés.

\section{REFERENCIAS}

1. Rodríguez RJ, Gattini C, Almeida G. El establecimiento de Sistemas de información en servicios de atención de la salud: Guía para el análisis de requisitos, especificación de las aplicaciones y adquisición. Pan American Health Organization. Washington, DC: PAHO, 1999.
2. Llanusa RS, et al. Las tecnologías de información y comunicación y la gestión del conocimiento en el sector salud. Revista Cubana de la salud Pública, 2005, vol. 31, no 3, p. 223-32.

3. Oviedo E, Fernández A. Tecnologías de la información y la comunicación en el sector salud: oportunidades y desafíos para reducir inequidades en América Latinay el Caribe. CEPAL, 2010.

4. Pressman R. Ingeniería de Software. Un enfoque práctico. McGraw- Hill, México, 2007.

5. Sommervville I. Ingeniería de Software. Pearson Educación, México, 2002.

6. Rojas Sola JI, Fernández Sora A, Serrano Tierz A y Hernández Díaz D. Una revisión histórica: desde el dibujo en ingeniería hacia la ingeniería del diseño, Revista Dyna., 78 (167), pp. 17-26, 2011.

7. Sunkel G, Trucco D. Nuevas tecnologías de la información y la comunicación para la educación en América Latina: riesgos y oportunidades. CEPAL, 2010.

8. Oviedo E, Fernández A. Tecnologías de la información y la comunicación en el sector salud: oportunidades y desafíos para reducir inequidades en América Latinay el Caribe. CEPAL, 2010.

9. Mildenberger $P$, Eichelberg $M$, Martin

E. Introduction to the DICOM standard. European radiology, 2002, vol. 12, no 4, p. 920-927.

10. Sideli R. et al. Adopting HL7 as a Standard for the Exchange of Clinical Text Reports. En Proceedings. Symposium on Computer Applications in Medical Care. American Medical Informatics Association, 1990. p. 226-229.

11. ASTM International - Standards and Publications https://www.astm.org/ 
Standard/standards-andpublications.html

12. WHO | ICD-10 online versions http://

www. who.int/classification

s/icd/ icdonlineversions/en/

13. Elkin PL. et al. Evaluation of the content coverage of SNOMED CT: ability of SNOMED clinical terms to represent clinical problem lists. En Mayo Clinic Proceedings. Elsevier, 2006. p. 741-748.

14. Donnelly K. SNOMED-CT: The advanced terminology and coding system for eHealth. Studies in health technology and informatics, 2006, vol. 121, p. 279.

15. Mcdonald CJ, et al. LOINC, a universal standard for identifying laboratory observations: a 5-year update. Clinical chemistry, 2003, vol. 49, no 4, p. 624-633.

16. Monteagudo PJ. SALVADOR, Carlos Hernández. Estándares para la historia clínica electrónica. Informes SEIS, p. 193, 2002.

17. Barrows HS. An overview of the uses of standardized patients for teaching and evaluating clinical skills. AAMC. Academic Medicine, 1993, vol. 68, no 6, p. 443-51.

18. ISO INTERNATIONAL ORGANIZATION FOR STANDARIZATION https://www.iso. org/

19. Crowley JR, William F., et al. Clinical research in the United
States at a crossroads: proposal for a novel public- private partnership to establish a national clinical research enterprise. Jama, 2004, vol. 291, no 9, p.1120-1126.

20. Frenk J. La salud de la población: Hacia una nueva salud pública. Fondo de Cultura Económica, 2016.

21. Hernández LR. Metodología de la investigación en ciencias de la salud. Ecoe Ediciones, 2008.

22. Cabieses B, Espinoza MA. La investigación traslacional y su aporte para la toma de decisiones en políticas de la salud. Revista Peruana de Medicina Experimental y Salud Pública, 2011, vol. 28, no 2, p. 288-297.

23. Betancourt BJ, Ramis AR, Mirabal NM. Investigación Traslacional y Transdisciplinaria en salud pública. Revista Cubana de la Salud Pública, 2014, vol. 40, no 2, p. 236-245.

24. Gómez D, Soto GN. Ilegalidad de la investigación genética en Colombia. Pensamiento Jurídico, 2007, no 18.

25. MODELO MEXICANO DE ARBITRAJE MÉDICO SECRETARÍA DE LA SALUD WWW.salud.gob.mx/unida des/cdi / documentos/DOCSAL7339.pdf

26. Valdés SR, Molina LJ, Solís TC. Aprender de lo sucedido. Análisis de las quejas presentadas ante la Comisión Nacional de Arbitraje Médico. Salud pública de México, 2001, 
Gutiérrez et. al. - Obstáculos y retos para el desarrollo de sistemas de información en el sector salud

vol. 43, no 5, p. 444-454.

27. INFORMADO, Consentimiento Válidamente. Comisión Nacional de Arbitraje Médico, 1a.Edición, México, DF, 2004, vol. 12.

28. Charette R. Análisis y administración del riesgo en Ingeniería de Software, McGraw- Hill, USA, 1989.

29. Sánchez MA. “Implicaciones éticas y socioeconómicas de las historias clínicas

electrónicas". Revista Informática Médica. Año 4, No. 10, $2002 . \quad$ www. informaticamedica.org.ar.

30. Peña GR. "Uso de la Planificación en el Desarrollo del Expediente Clínico Electrónico", Tesis de Maestría en Ingeniería, Universidad Nacional de México (UNAM), 2003. 